\title{
Electron beam and rf characterization of a low-emittance $X$-band photoinjector
}

\author{
D. J. Gibson and F. V. Hartemann \\ Institute for Laser Science and Applications, Physics and Advanced Technologies Directorate, \\ Lawrence Livermore National Laboratory, Livermore, California 94550 \\ E. C. Landahl, A. L. Troha, and N.C. Luhmann, Jr. \\ Department of Applied Science, University of California, Davis, California 95616 \\ G.P. Le Sage \\ Defense Sciences Engineering Division, Engineering Directorate, Lawrence Livermore National Laboratory, \\ Livermore, California 94550 \\ C. H. Ho \\ Synchrotron Radiation Research Center, Hsinshu 30077, Taiwan
}

(Received 17 April 2001; published 26 September 2001)

\begin{abstract}
Detailed experimental studies of the first operation of an $X$-band $(8.547 \mathrm{GHz}) \mathrm{rf}$ photoinjector are reported. The rf characteristics of the device are first described, as well as the tuning technique used to ensure operation of the $1 \frac{1}{2}$-cell rf gun in the balanced $\pi$-mode. The characterization of the photoelectron beam produced by the rf gun includes: measurements of the bunch charge as a function of the laser injection phase, yielding information about the quantum efficiency of the $\mathrm{Cu}$ photocathode $\left(2 \times 10^{-5}\right.$ for a surface field of $100 \mathrm{MV} / \mathrm{m})$; measurements of the beam energy $(1.5-2 \mathrm{MeV})$ and relative energy spread $\left(\Delta \gamma / \gamma_{0}=1.8 \pm 0.2 \%\right)$ using a magnetic spectrometer; measurements of the beam $90 \%$ normalized emittance, which is found to be $\varepsilon_{n}=1.65 \pi \mathrm{mm}$ mrad for a charge of $25 \mathrm{pC}$; and measurements of the bunch duration $(<2 \mathrm{ps})$. Coherent synchrotron radiation experiments at Ku-band and Ka-band confirm the extremely short duration of the photoelectron bunch and a peak power scaling quadratically with the bunch charge.
\end{abstract}

DOI: 10.1103/PhysRevSTAB.4.090101

PACS numbers: 29.25.Bx, 41.75.Ht, 41.75.Lx, 41.60.-m

\section{INTRODUCTION}

Compact, high-gradient $(>100 \mathrm{MeV} / \mathrm{m})$ rf photoinjectors capable of producing relativistic $(>5 \mathrm{MeV})$ electron beams with extremely low emittance $(<1 \pi \mathrm{mm} \mathrm{mrad})$, high charge $(1 \mathrm{nC})$, and subpicosecond duration are currently being developed [1-5] for a variety of applications ranging from the Next Linear Collider (NLC) to compact, prebunched free-electron masers [6]. Such electron accelerators will also have a number of important applications in biomedical imaging and therapy and in drug manufacturing. For example, $33.1 \mathrm{keV} \mathrm{x}$ rays can be used for high-contrast angiography [7] by taking advantage of the K-edge of iodine. Likewise, at $1 \AA(12 \mathrm{keV})$, protein crystallography is revolutionizing the pharmaceutical industry by allowing the design of new drugs, as exemplified by the protease inhibitors used to decrease the viral load in HIV patients [8]. This type of radiation can be produced by Compton scattering [9] using a high-quality electron beam and a tabletop terawatt laser for an advanced $\mathrm{x}$-ray source. Compactness, low cost, and turnkey operation are highly desirable for such sources, especially in a clinical environment, and the development of new technologies for electron acceleration will assist in attaining that goal. Extreme ultraviolet and soft $\mathrm{x}$-ray lithography also has tremendous potential advantages for the microchip industry. Finally, these high-gradient electron accelerators will also be extremely useful as basic research tools in universities, industry, and government laboratories.

Within this context, we are developing an $X$-band (8.547 GHz) rf gun, based on the $1 \frac{1}{2}$-cell, $S$-band Brookhaven National Laboratory (BNL) design [10]. A detailed theoretical and computational study of the scaling of $\mathrm{rf}$ injector beam performance with frequency has been performed [11], with the conclusion that $X$-band represents an optimum balance between beam quality and accelerated charge and should reach very high brightness. These higher frequency rf photoinjectors offer the possibility of operating at larger peak accelerating fields, thus alleviating the detrimental effects of space charge, which scale as $\gamma^{-1}$, where the identity $\gamma-1=e v / m_{0} c^{2}$ defines the relativistic mass factor $\gamma$ of the electrons at the potential $v$. For electrons, the quantity $m_{0} c^{2} / e=0.511 \mathrm{kV}$.

This paper is organized as follows: The experimental setup, including the $X$-band rf system and the UV photocathode laser illumination system, as well as the timing and synchronization subsystems, is discussed in Sec. II. Section III focuses on the characterization of the photoelectron beam produced by the $X$-band gun, including measurements of the bunch charge as a function of the laser injection phase and the Schottky effect, evaluation of the beam energy and relative energy spread using a 
magnetic spectrometer, measurements of the beam emittance, an estimate of the bunch duration using both a streak camera and coherent synchrotron radiation, and a study of the timing jitter between the laser and rf accelerating fields. Finally, conclusions are drawn in Sec. IV.

\section{EXPERIMENTAL SETUP}

\section{A. $X$-band rf system}

To ensure proper synchronization between the laser and rf systems, two main options exist: one can either use an external rf clock, which then drives both the high-power rf system energizing the rf gun and the mode-locked laser oscillator, or the rf signal can be derived from the light pulse train produced by a self-mode-locked laser oscillator. Because of its considerably simpler implementation, the latter option was used in these experiments; its jitter performance is discussed in Secs. IID and IIIF. A photodiode monitoring the $79.138 \mathrm{MHz}$ oscillator pulse train sends this signal, after proper filtering, to a phase-locked dielectric resonance oscillator (PDRO), which increases the frequency by a factor of 108 , producing a $8.547 \mathrm{GHz}$ signal. The PDRO has an input frequency range of $\pm 50 \mathrm{MHz}$, yielding the frequency tuning required for proper conditioning of the high-power rf gun. This signal is then sent to a $1 \mathrm{~kW}$ traveling wave tube amplifier (TWTA), followed by a Stanford Linear Accelerator Center (SLAC) SL-3 klystron [12] capable of producing up to $19 \mathrm{MW}$ of rf power in 1-2 $\mu$ s square pulses, at up to a $50 \mathrm{~Hz}$ repetition rate. The power is measured by using a precision thermistor head for the average power and a fast $(<600 \mathrm{ps}$ rise time) $\mathrm{rf}$ diode for determination of the pulse duration. For the present experiments, the klystron runs at $20 \mathrm{~Hz}$ with a $250 \mathrm{~ns}$ rf pulse, to allow for higher gradients in the gun without rf breakdown problems.

The heart of the rf system is a high- $Q$ (4 274 measured), $X$-band rf gun. The brazed gun is shown in Fig. 1(a), while a schematic of the entire experimental setup is presented in Fig. 1(b). The $1 \frac{1}{2}$-cell design yields good rf and electron beam characteristics, as summarized in Table I; however, the $\pi$-mode magnetic coupling scheme employed in this design is quite sensitive to mechanical tolerances. In particular, achieving critical coupling in the structure requires
TABLE I. $\quad X$-band $\mathrm{rf}$ gun design parameters.

\begin{tabular}{lc}
\hline \hline Beam energy & $5.7 \mathrm{MeV}$ \\
Coupled rf power & $16 \mathrm{MW}$ \\
Bunch charge & $0.1 \mathrm{nC}$ \\
Normalized emittance & $0.7 \pi \mathrm{mm} \mathrm{mrad}$ \\
Energy spread & $0.25 \%$ \\
Bunch duration & $0.9 \mathrm{ps} \mathrm{FWHM} \mathrm{Gaussian}$ \\
Laser spot size & $0.67 \mathrm{~mm} \mathrm{FWHM} \mathrm{Gaussian}$ \\
Optimum injection phase & $65^{\circ}$ \\
\hline \hline
\end{tabular}

a very high degree of precision in the design and fabrication of the coupling holes between the WR-90 waveguide and the half and full cells; in practice, the small mechanical shifts induced by the brazing process require a postbrazing tuning mechanism to compensate for these effects. In the present case, a custom tuning post was fabricated by SLAC to ensure that the peak of the gun resonance is properly correlated with the maximum of the SL-3 rf power tuning curve. This tuning of the rf gun coupling is supplemented by two independent tuners for the half and full cells, and the overall resonant frequency of the system can be temperature tuned once balanced, $\pi$-mode critical coupling is achieved.

The main steps of the gun tuning procedure are as follows: (i) using a network analyzer, the full cell is tuned slightly above the desired operation frequency (the exact design frequency is reached by temperature tuning the structure), (ii) we merge the half-cell resonance for balanced $\pi$-mode operation, (iii) the operation frequency is reached by heating the structure. The reflection from the $X$-band gun, after the tuning procedure described above, is shown in Fig. 2(a). In this case, the resonant frequency is $f=8.547 \mathrm{GHz}$, the reflected wave is $-74.266 \mathrm{~dB}$ below the input signal, and the $-3 \mathrm{~dB}$ bandwidth of the resonance is $4 \mathrm{MHz}$, which corresponds to a $Q$ value of 4274 . The gun temperature-tuning curve is shown in Fig. 2(b), and is found to be in excellent agreement with the theoretical prediction based on a cylindrical pillbox model of the gun [13]. The $\mathrm{TM}_{010}$ resonant frequency is given by $f_{010}=\frac{c}{2 \pi} \frac{\chi_{01}^{\prime}}{a}$, where $a$ is the cavity radius and $\chi_{01}^{\prime}$ is the first root of the zeroth-order Bessel function; using the linear coefficient of thermal expansion for copper,
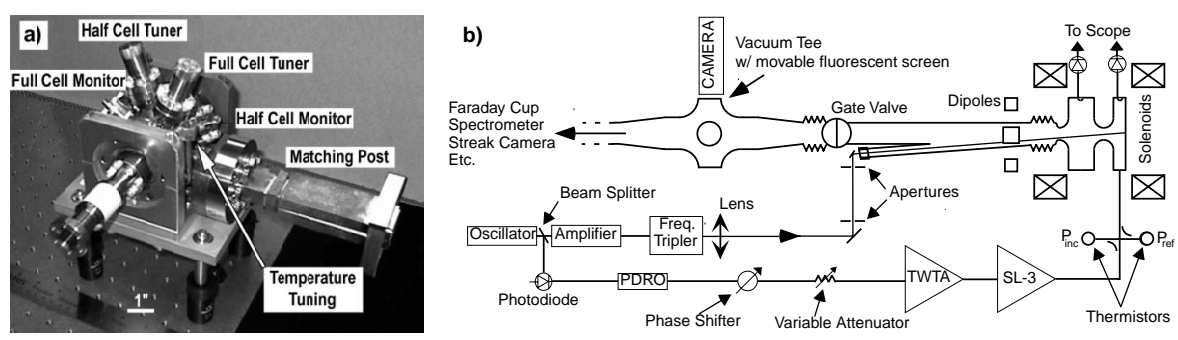

FIG. 1. (a) Photograph of the $X$-band rf gun, showing the tuning and monitoring features, and (b) a schematic of the experimental setup. 


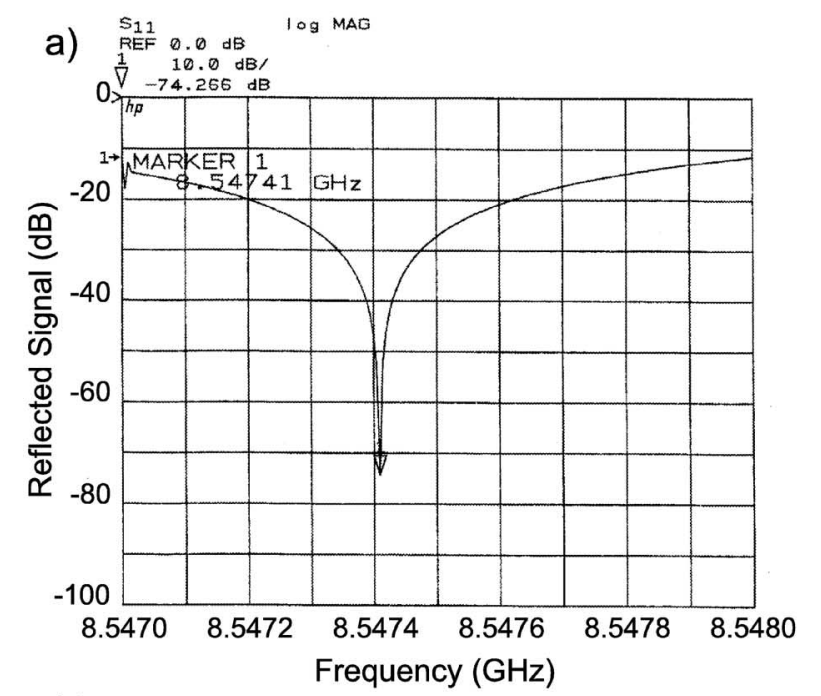

b)

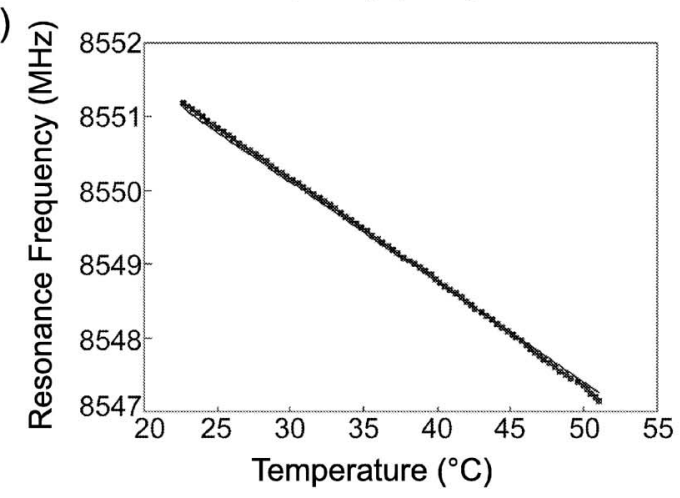

FIG. 2. (a) Gun power reflection as a function of frequency. The horizontal axis runs from 8.547 to $8.548 \mathrm{GHz}$ with each division being $100 \mathrm{kHz}$, and the vertical axis runs from 0 to $-100 \mathrm{~dB}$ with each division being $10 \mathrm{~dB}$. (b) Gun resonance frequency as a function of temperature. The line is the theoretical prediction [Eq. (1)] and the points are measured values.

$\alpha=1.6 \times 10^{-6}{ }^{\circ} \mathrm{C}^{-1}$, the slope of the frequency shift can be calculated to be $-136 \mathrm{kHz} /{ }^{\circ} \mathrm{C}$ as follows:

$$
\Delta f=\frac{d f}{d a} \frac{d a}{d T} \Delta T=-\alpha f_{010} \Delta T .
$$

The corresponding linear fit is also shown in Fig. 2(b), and the agreement is excellent.

The next experimental step involves repeating these measurements in situ, with the full rf signal. Magnetic pickup loops are located within the half and full cells and are designed with very low coupling parameters in order to avoid perturbing the rf fields in the accelerating structure and causing rf breakdown. Their calibration procedure is straightforward: the gun is tuned to obtain a hard resonance in either the half or the full cell, and the power coupled into the structure is obtained by measuring both the incident and reflected power. The crystal detectors are calibrated at the same frequency with a $\mathrm{cw}$ rf source, a frequency counter, and a power meter using a precision thermistor head. The half-cell monitor coupling parameter is thus found to be $-57.4 \mathrm{~dB}$, while the full

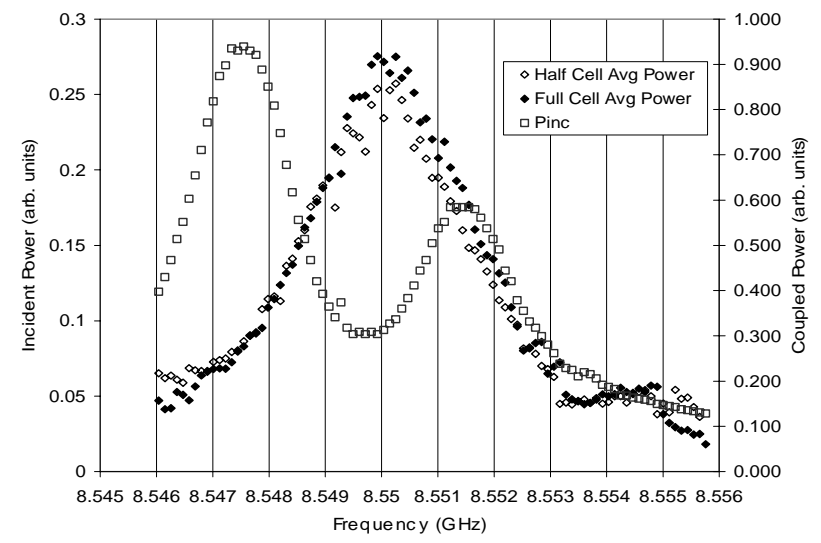

FIG. 3. Power incident upon the gun $\left(P_{\text {inc }}\right)$, and the power in the half and full cells of the gun as measured by the monitor probes and normalized to the incident power.

cell monitor couples $-46.9 \mathrm{~dB}$ of the incident power. The power coupled in the half and full cells is shown in Fig. 3 as a function of the frequency and normalized to the input power. The difference in power between the half and full cell corresponds to a $3 \%$ variation in terms of the rf fields, and shows balanced excitation of the gun. At this point, the most critical remaining step is to verify that the mode excited in the gun is the proper accelerating $\pi$-mode. The experimental setup is shown in Fig. 4(a). First, an absolute calibration of the $X$-band interferometer is performed by splitting the incident power from the PDRO with a tee [Fig. 4(b)]: the power on each arm is first balanced with a precision attenuator, and the mixer response is measured as a function of the phase shifter settings, as shown in Fig. 5.

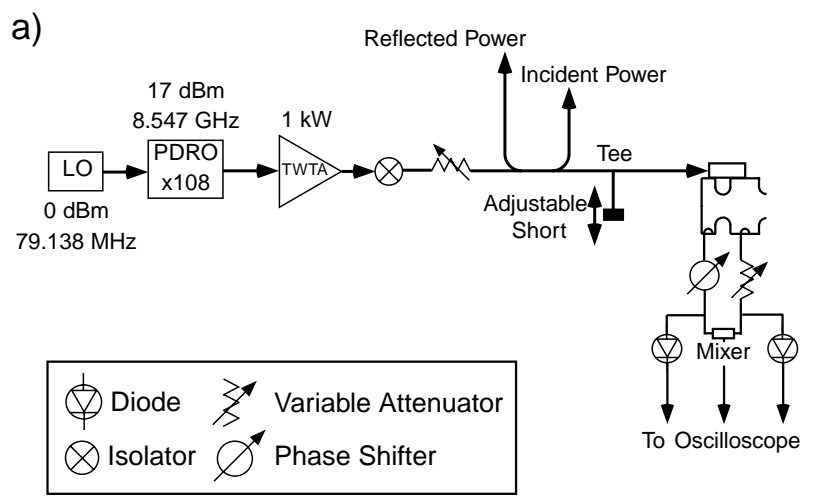

b)

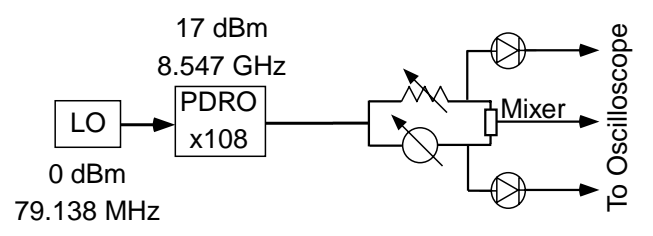

FIG. 4. Schematic of (a) the interferometer setup used to check the relative phases of the half and full cells of the gun, and (b) the setup used to calibrate the interferometer. 


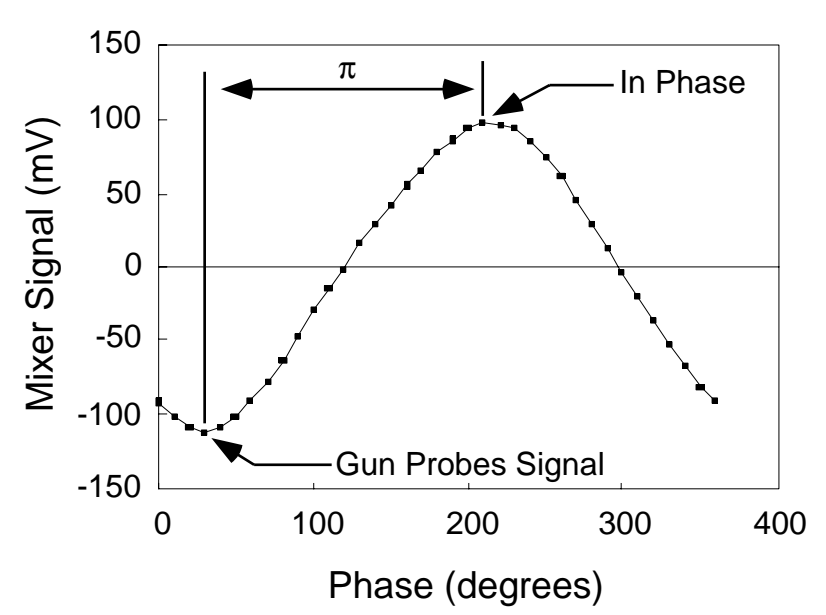

FIG. 5. The calibration curve of the interferometer, showing the mixer signal as a function of phase shifter phase setting. Setting the phase shifter to give a maximal result during calibration yields a minimal result when measuring the gun, indicating a $\pi$-phase shift between the two cells.

The frequency chosen for the calibration corresponds to the frequency at which critical coupling is achieved. We then set the interferometer at the phase giving the maximum positive output signal $\left(210^{\circ}\right.$, in this case), and replace the tee with the gun probe outputs. Note that the orientation of the loops is carefully controlled for these phase measurements; in addition, both probes have exactly the same microwave path length. Again, the interferometer power is balanced, and we observe a mixer output signal with maximum amplitude and negative polarity; this shows that the relative phase of the gun field monitors is shifted by $180^{\circ}$, as compared to the reference signals provided by the tee in the calibration described above [see Fig. 4(b)]. Thus, balanced $\pi$-mode excitation has been demonstrated; in addition, we have varied the tuning of the half and full cells and the operation frequency around these parameters and found that we could not excite the 0-mode, which indicates that $\pi$-mode coupling conditions are quite robust, as designed by BNL. Two solenoids, aligned to yield antiparallel magnetic fields and a null on the photocathode, are used to extract the beam from the gun.

\section{B. UV photocathode laser}

The laser system used to illuminate the photocathode is a commercial chirped-pulse amplification system, consisting of an oscillator, a grating stretcher, a regenerative amplifier, a grating compressor, and a frequency tripler. The system is based entirely on diode pumped solid-state laser technology, which provides the stability needed to illuminate the photocathode reliably. The oscillator is a Ti:sapphire (Ti: $\mathrm{Al}_{2} \mathrm{O}_{3}$ )-based FemtoSource Pro commercial system from Femtolasers Produktions $\mathrm{GmbH}$, capable of producing pulses as short as $10 \mathrm{fs}$ via Kerr-lens mode locking [14]. These pulses are produced by the technique of mirror-dispersion control [15], where the dispersion introduced by the thin Ti: $\mathrm{Al}_{2} \mathrm{O}_{3}$ crystal is compensated for in the dielectric coating on the mirrors; the layout of this system is shown in Fig. 6. This oscillator is pumped by a $5 \mathrm{~W}$ $\mathrm{cw}, 532 \mathrm{~nm}$ beam produced by the intracavity frequencydoubled, diode-pumped Nd: $\mathrm{YVO}_{4}$ Millennia V laser from Spectra-Physics. Since $10 \mathrm{fs}$ pulses are not needed for this application, are difficult to create and maintain, and will be unavoidably lengthened in this particular amplifier, the oscillator is operated without the final compensation optics, yielding a slightly chirped pulse that is $15 \mathrm{fs}$ in duration. The pulse duration is measured with an interferometric autcorrelator where the pulse is interfered with a time-delayed copy of itself in a nonlinear optical medium. As the time delay is changed, an interference pattern is produced corresponding to the pulses' second harmonic autocorrelation [Fig. 7(a)], and the number of fringes in this pattern, along with the wavelength of the light, can be used to determine the temporal pulse width. This pulse has a bandwidth of $>70 \mathrm{~nm}$ centered around $800 \mathrm{~nm}$ [Fig. 7(b)]. The oscillator was assembled with the output coupler mounted on a translation stage; using this setup, the repetition rate of the oscillator can easily be adjusted between 79.120 and $79.155 \mathrm{MHz}$ without interfering with the Kerr-lens mode-locking process. In order to properly synchronize the laser with the rf in the gun, the oscillator cavity length was adjusted to produce a repetition rate of $79.138 \mathrm{MHz}$ (the 108th subharmonic of the optimal gun operating frequency of $8.457 \mathrm{GHz}$ ). The resulting oscillator output pulse train is shown in Fig. 7(c).

The output of the oscillator is then coupled into a sub$50 \mathrm{fs}, 1 \mathrm{kHz}$ Spitfire system from Positive Light, which includes the grating stretcher, $\mathrm{Ti}_{\mathrm{Al}} \mathrm{Al}_{2} \mathrm{O}_{3}$ regenerative amplifier, and grating compressor. The regenerative amplifier crystal is pumped by a $7 \mathrm{~W}$ average power, $1 \mathrm{kHz}$, $Q$-switched, diode-pumped Nd:YLF Evolution laser from Positive Light. This system, as currently configured, selects a pulse from the $79.138 \mathrm{MHz}$ pulse train and amplifies it to an energy of $0.4 \mathrm{~mJ}$, with a duration of $60 \mathrm{fs}$

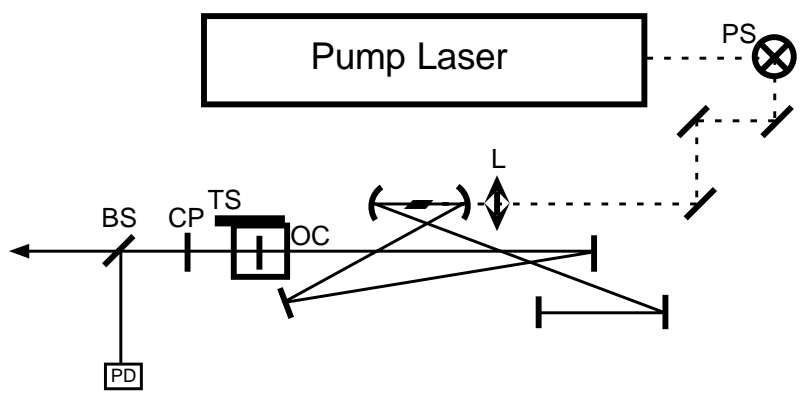

FIG. 6. The Ti: $\mathrm{Al}_{2} \mathrm{O}_{3}$ laser oscillator layout. All components are mirrors unless labeled. PS: periscope; L: focusing lens; OC: output coupler; CP: compensating plate; BS: beam splitter; PD: photodiode; and TS: translation stage. The solid line is the $800 \mathrm{~nm}$ oscillator beam, the dashed line is the $532 \mathrm{~nm}$ pump beam. 

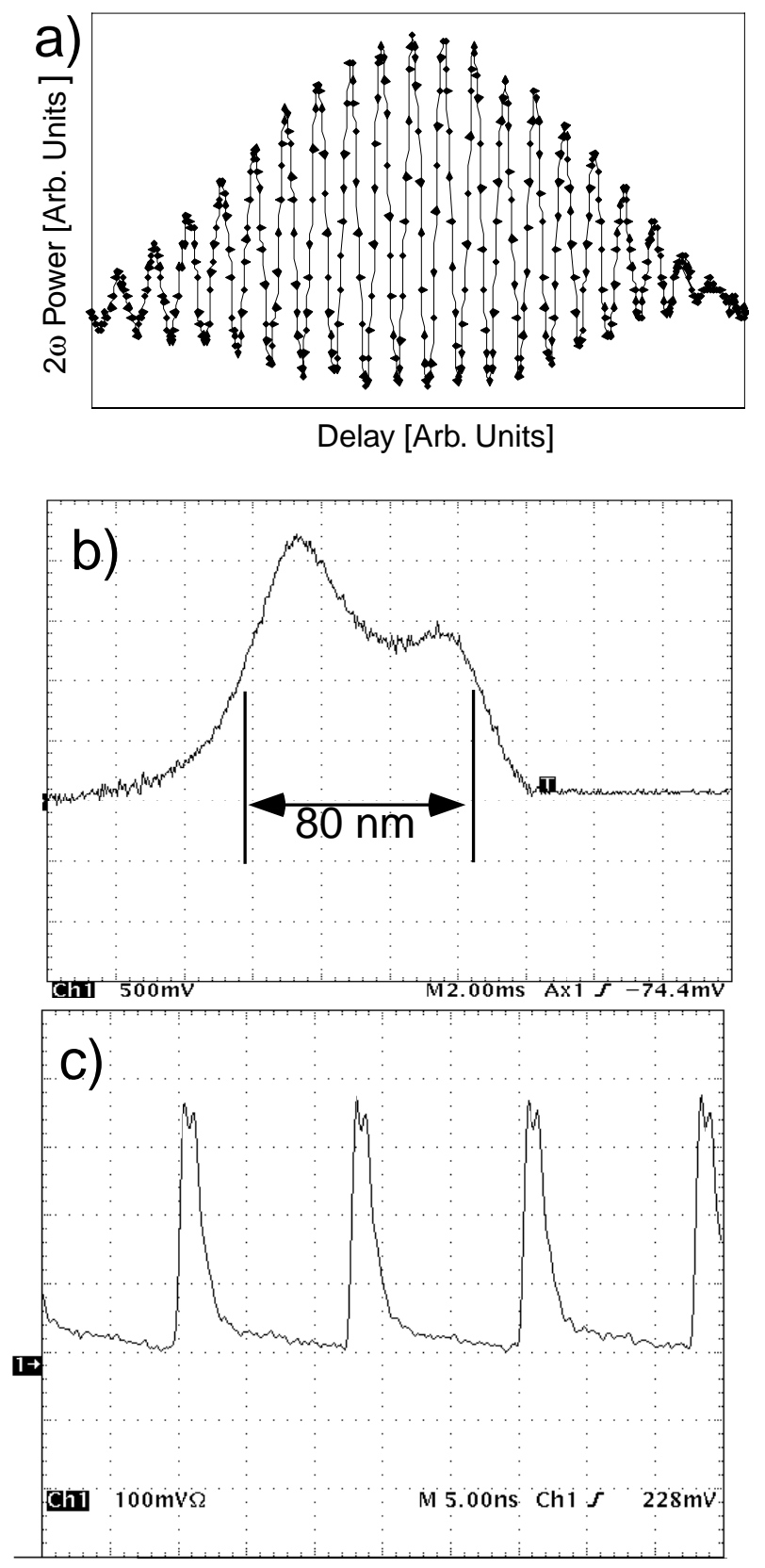

FIG. 7. (a) Autocorrelation trace, (b) spectrum, and (c) output pulse train as measured on photodiode of the Ti: $\mathrm{Al}_{2} \mathrm{O}_{3}$ oscillator (5 ns/div).

and an overall repetition rate of $1 \mathrm{kHz}$. The output pulse is longer than the input pulse because the spectrally spread out input beam in the stretcher is wider than the retroreflecting mirror in the system and therefore the bandwidth is clipped down to $25 \mathrm{~nm}$, resulting in a larger minimum pulse length as predicted by Fourier theory. Figure 8 shows the output spectrum of the Spitfire, the corresponding autocorrelation trace, as measured with the single-shot autocorrelator also produced by Positive Light, and the output pulse as measured with a photodiode. The second small pulse corresponds to a portion of the first pulse that was not completely switched out of the regenerative
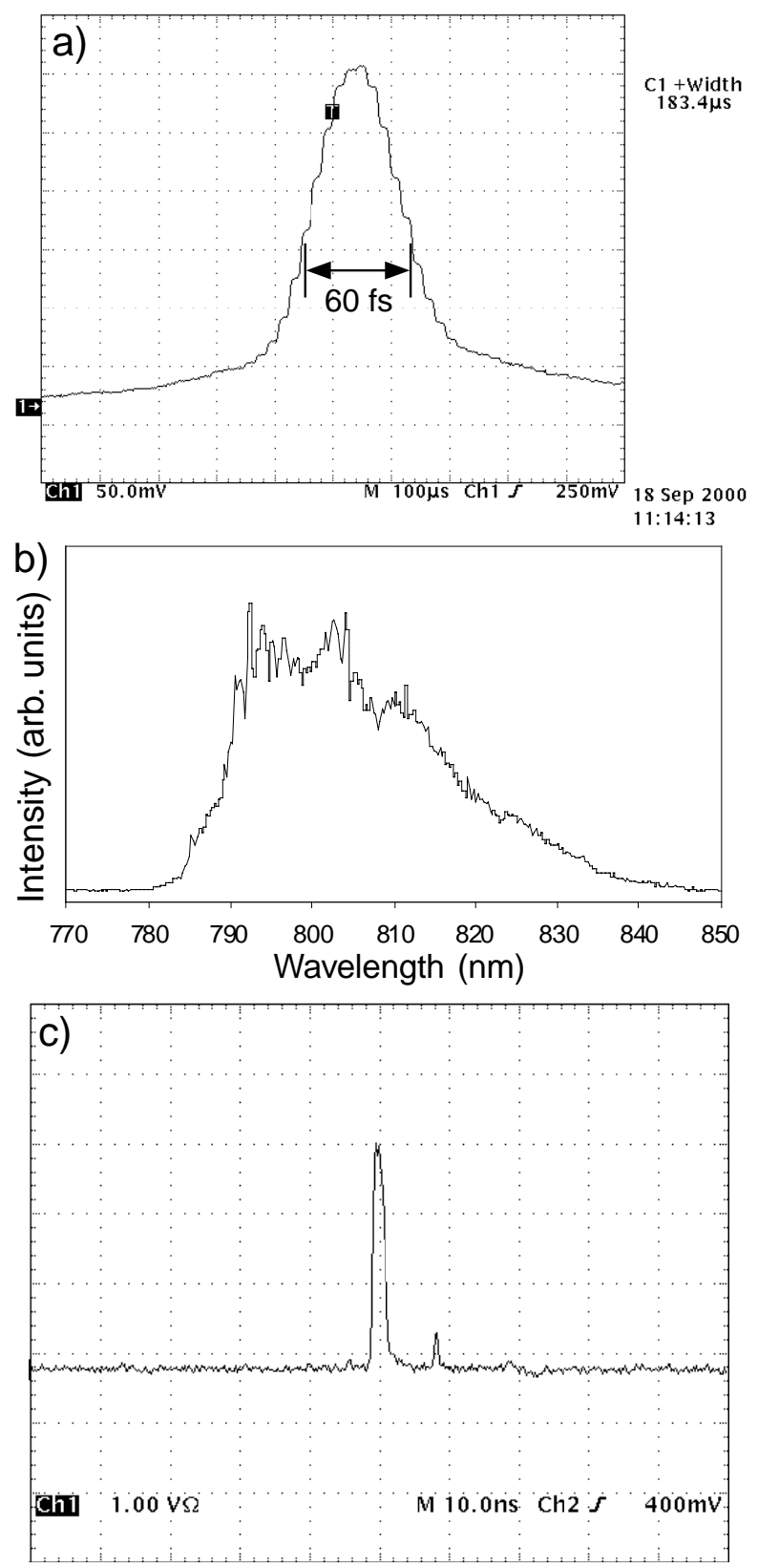

FIG. 8. (a) Autocorrelation trace, (b) spectrum, and (c) temporal profile as measured on photodiode of one pulse as it exits the regenerative amplifier (10 $\mathrm{ns} /$ div).

cavity after amplification and made an extra trip through cavity; this pulse does not interfere with the operation of the photoinjector, as it is frequency-tripled with very low efficiency.

The amplified pulse is then directed into a frequency tripler produced by Spectra-Physics. This specific tripler design uses two $\beta$-barium borate crystals for second- and third-harmonic generation. This converts the incoming IR beam to $266 \mathrm{~nm}$, which corresponds to the work function of copper at low fields. To compensate for the walk off between the IR and the blue in the first crystal, a strongly birefringent crystal is used, which synchronizes the two 
beams temporally before injection in the tripling crystal. Using this system, UV pulses with $6 \mu \mathrm{J}$ of energy are routinely produced. Because of fluctuations in the duration of the output pulse of the Spitfire, the intensity of the UV pulse varied between 5 and $7 \mu \mathrm{J}$ on a time scale of a few seconds. These fluctuations seem to be related to instabilities, e.g., from air currents, in the compressor section of the Spitfire.

The UV beam is then injected onto the photocathode at 3 degrees from normal incidence using a custom-made high-vacuum "Y" piece attached to the output face of the gun [see Fig. 1(b)]. The straight arm of the $\mathrm{Y}$ is the photoelectron beam line, and the 3-degree arm is sealed with a Suprasil window, which has good transmission at $266 \mathrm{~nm}$. In order to align the laser down the beam pipe properly, a lamp was placed against the beam line to illuminate the copper photocathode, which diffusely reflected some of the light down the Y. Two apertures were then placed such that the cathode could clearly be seen through both of them, and the laser was subsequently passed through the apertures. Following this procedure, and adjusting the rf phase, immediately produced the photoelectron beam.

\section{Beam line}

The beam line used for the experiment is shown in Fig. 1(b). Following the extraction solenoids mentioned above is a pair of orthogonal dipole magnets that are used to steer the beam and center it on the detectors. The beam passes through a gate valve which can be closed to allow easy changes to the beam line to accommodate different measurements. For most of the measurements, the gate valve was followed by a vacuum tee with a fluorescent screen which could be moved in and out of the beam path. Off to one side was a video camera used to capture the fluorescence from the screen. Different experimental setups were deployed following the tee: to measure quantum efficiency, a $50 \Omega$-matched Faraday cup is used; for the beam energy and energy spread measurements, a magnetic spectrometer is attached. A quartz window placed at the end of the beam line allows the use of either a streak camera for beam duration and timing jitter measurements or an rf detector system (as discussed in Sec. III G) to study coherent synchrotron radiation. For the emittance measurement, another vacuum tee with a tantalum plate with various sized holes and a $1 \mathrm{~m}$ beam pipe was placed between the gate valve and the fluorescent screen, as discussed in Sec. III D.

\section{Timing}

In order to produce a photoelectron beam reliably, it is essential to illuminate the photocathode within a very narrow temporal window, extending about $1^{\circ}$ off the optimal rf phase. At the operating frequency of $8.547 \mathrm{GHz}$, this requirement translates to a timing accuracy of approximately $0.3 \mathrm{ps}$. The overall timing system used is shown in Fig. 9. The black boxes represent laser components and the white

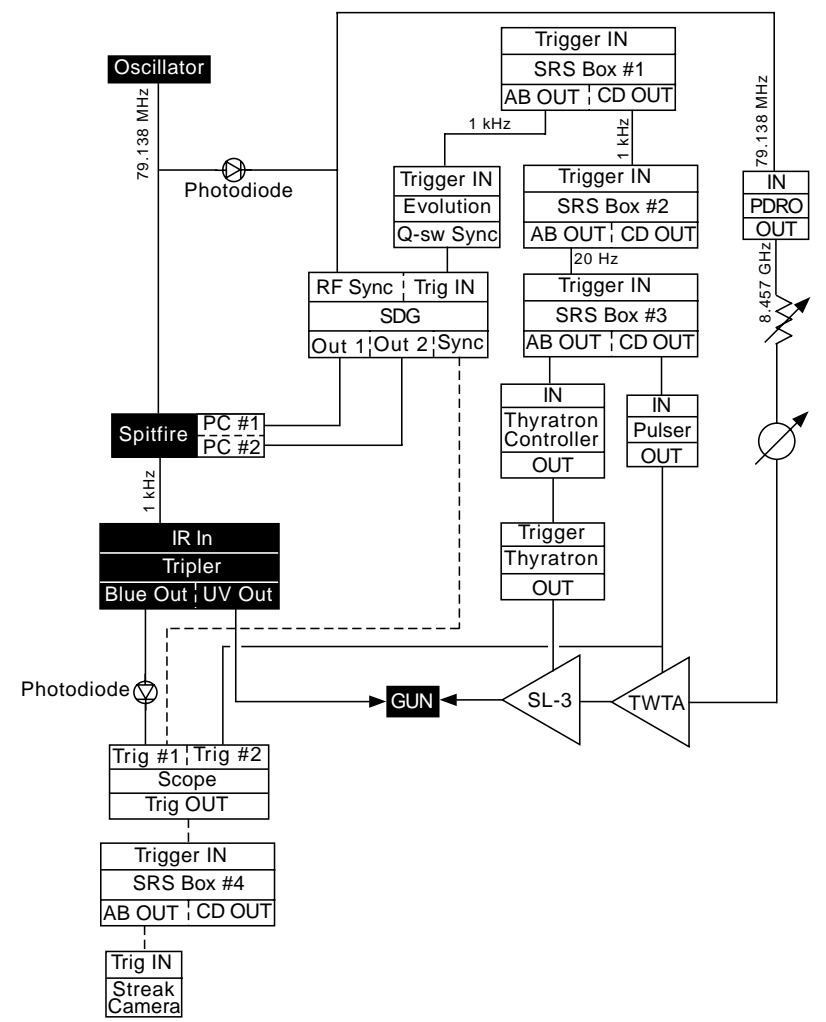

FIG. 9. Diagram of trigger, laser, and rf signals in the entire experimental setup. Black boxes are laser elements, white boxes are electronic elements. SRS boxes are Stanford Research Systems DG535 delay generators, SDG is the Positive Light synchronization and delay generator, and PC is Pockels cell. See text for full description.

boxes represent electrical components. This system solves the three main problems of timing: (i) producing the photoelectrons at the proper rf phases, (ii) properly timing the laser and rf fields in the gun, and (iii) measuring quantities associated with a $20 \mathrm{~Hz}$ system that are synchronized to a $1 \mathrm{kHz}$ event.

As mentioned in the Introduction, one method that can be used to lock the phase of the rf to the pulse train of the laser is to run a master clock and use feedback to adjust the oscillator to keep the output in phase with the clock. Another possibility, which does not require any feedback and is considerably easier to implement, is used in this experiment: the $79.138 \mathrm{MHz}$ pulse train from the oscillator is sampled with a photodiode, this signal is then fed into the PDRO, which raises the frequency by a factor of 108 yielding a $8.547 \mathrm{GHz}$ signal that is precisely locked in phase with the laser pulses. This signal then goes through a variable attenuator to control the power in the gun and a phase shifter to adjust and optimize the timing of the laser pulse and the rf phase. This signal is subsequently gated and amplified by both the TWTA and the klystron before injection into the gun.

In order to ensure that the UV laser pulse arrives in the gun while the TWTA and klystron are energized, a master clock is used. The $1 \mathrm{kHz}$ timing signal originates from 
a Stanford Research Systems pulse and delay generator DG535 (an "SRS box") which is capable of outputting two different, arbitrary pulses with a specified precision of 5 ps. The SRS box (hereafter referred to as SRS box \#1) is free running at $1 \mathrm{kHz}$. One of the two output pulses is used to trigger the $Q$-switching of the regenerative amplifier pump laser. When $Q$-switching occurs, a pulse is sent to a synchronization and delay generator (SDG). The SDG compares the $1 \mathrm{kHz} Q$-switch signal to the $79.138 \mathrm{MHz}$ photodiode signal mentioned above. When the pump laser fires, the next pulse from the photodiode triggers three output signals with user-defined delays. Two of these signals are used to control the two Pockels cells in the regenerative amplifier, and the third can be used to trigger an oscilloscope.

The second output pulse from SRS box \#1 is sent to SRS box \#2, which divides the $1 \mathrm{kHz}$ signal down to $20 \mathrm{~Hz}$; the $20 \mathrm{~Hz}$ signal then controls SRS box \#3. The first pulse from the third box triggers the thyratron controller, which fires the thyratron and thereby determines when the klystron electron beam is on. The second pulse from SRS box \#3 triggers a pulse generator, which is used to create the pulse that gates the TWTA and determines the timing and duration of the rf signal in the klystron and $\mathrm{rf}$ gun. Therefore, by adjusting SRS box \#3, the rf signal can be optimally synchronized relative to the klystron electron beam, and by adjusting SRS box \#1 the laser can be properly timed relative to the $\mathrm{rf}$ in the gun.

In order to take measurements of the photoelectron beam, a trigger for the oscilloscope that is synchronized to the laser is required; however, not all of the UV laser pulses (at $1 \mathrm{kHz}$ ) will occur concurrently with accelerating fields in the gun (at $20 \mathrm{~Hz}$ ). Therefore, a logic gate is used on the oscilloscope to trigger only when there is both a laser pulse and a gate pulse to the TWTA. This trigger signal is also output from the scope and used to fire the streak camera for measurements. An additional SRS box allows fine tuning of the timing of the streak image.

Once the timing of the system is properly set, the shotto-shot timing jitter needs to be studied. This involves both a measurement of the jitter between events and the master clock and the relative jitter between two events that require simultaneity. There are two pairs of events for which the amount of jitter is crucial: the arrival of the UV laser pulse and the timing of the rf pulse in the gun, and the arrival of the UV laser pulse and the phase of the rf in the gun. The former is easily studied by comparing the signals on an oscilloscope (see Fig. 10). There is about 50 ns of jitter in the arrival of the laser relative to the rf pulse. This is most likely a result of the random selection of one of four pulses (spaced $12 \mathrm{~ns}$ apart) by the SDG in starting the timing cycle of the regenerative amplifier. However, since the gun is filled with a fairly uniform rf power for approximately $150 \mathrm{~ns}$, that jitter is not very significant. The latter form of jitter (laser timing versus rf phase) is more difficult to measure and is discussed in Sec. IIIF.

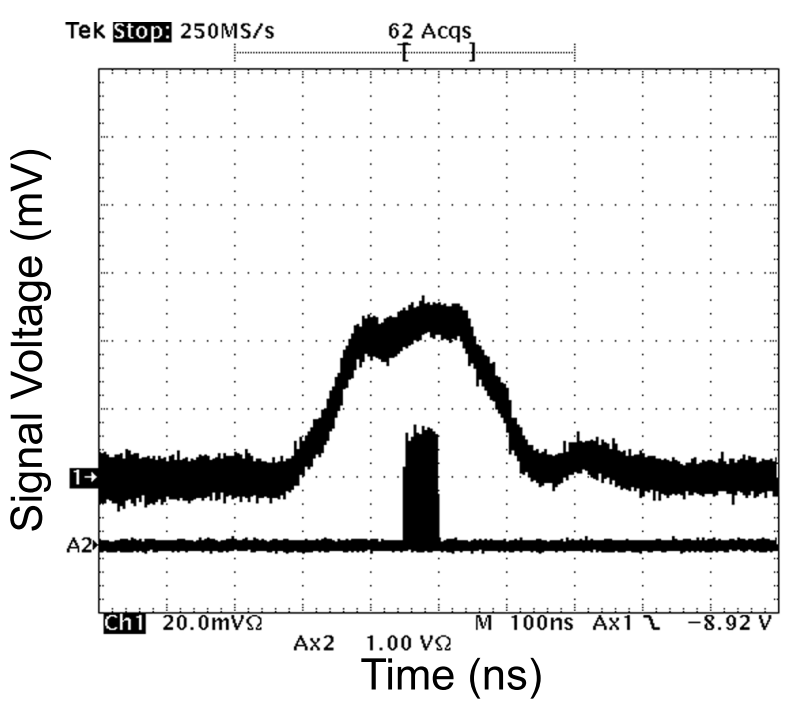

FIG. 10. Several overlain traces demonstrating the extent of shot-to-shot laser arrival timing jitter. The upper trace is the full cell power measured by the monitoring probe, and the lower trace is the signal from a photodiode measuring the laser. The horizontal scale is $100 \mathrm{~ns} / \mathrm{div}$, and the vertical scale is in arbitrary units.

\section{PHOTOELECTRON BEAM CHARACTERIZATION}

The photoelectron beam is optimized by examining Faraday cup measurements and the image of the beam on a phosphor screen; adjustments can be performed by varying the laser injection phase, the rf power energizing the gun, or the magnet focusing strength. A current of $25-50 \mathrm{~A}$ is used in the extraction solenoids mentioned above, which corresponds to a magnetic field strength in the 1-2 kG range. Additionally, the amount of power in the gun chosen for measurements is 1-2 MW, which was selected because, at this level, a high-quality electron beam is produced, and dark current is minimal.

In measuring the various relevant quantities of the electron beam, a fairly large amount of random fluctuation was noticed. It is believed that most of this was due to instabilities in the intensity and pointing of the laser system. Additionally, the timing jitter between the rf phase and laser injection was larger than the optimal 0.3 ps range, which also degraded the shot-to-shot beam quality significantly. In gathering these data, with the exception of the charge and Schottky values, single-shot measurements were performed to capture some of the cleaner events; therefore these numbers represent an optimal beam which was not continuously generated.

\section{A. Dark current}

Before discussing the photoelectron beam characteristics in detail, a few words concerning dark current are in order. At power levels in excess of $100 \mathrm{~kW}$, dark current was detected with the Faraday cup. The dark current is a 
strong function of the electric field at the photocathode surface and (from the Fowler-Nordheim equation [16]) varies exponentially with the electric field at the photocathode. The dependence of the field-emission current with the applied field at the cathode appears very clearly in Fig. 11: during the ramp up of the rf power in the gun, the exponentiation of dark current is evident; furthermore, the small rf power fluctuations observed in the gun are translated into comparatively larger variations in the dark current.

In practice, one must carefully rf- and vacuum-condition the photoinjector to help minimize field emission, which produces a weak beam that can generate background $x$ rays via bremsstrahlung and erode various surfaces in the rf structure through direct electron bombardment, as well as secondary electron emission and potential avalanche breakdown initiated by the dark current. Furthermore, in more complex structures, the large number of emitting surfaces subjected to high rf fields can result in the emission of different dark current beams, which can severely limit the operation of the device and the detection and characterization of the photoelectron beam. For the experiments described here, however, dark current proves to be a minimal problem when compared with breakdown at the aforementioned matching post required to achieve critical coupling, which limited the power coupled into the gun to approximately $3 \mathrm{MW}$ with a $150 \mathrm{~ns}$ flattop rf pulse; this corresponds to an average accelerating gradient of $80 \mathrm{MeV} / \mathrm{m}$ and a peak rf field at the cathode surface, $E_{0}=183 \mathrm{MV} / \mathrm{m}$. Typical operation was achieved in the 1-2 MW range, corresponding to cathode fields of 106 to $150 \mathrm{MV} / \mathrm{m}$, with output beam energies varying between 1.425 and $2 \mathrm{MeV}$.

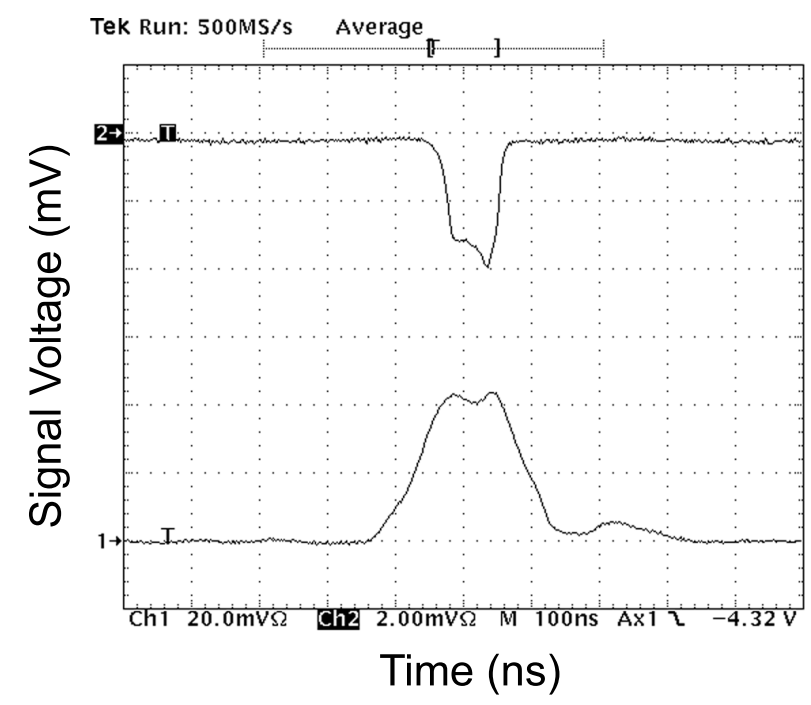

FIG. 11. Faraday cup measurements of dark current. The upper trace is the dark current signal with a cathode gradient of $125 \mathrm{MeV} / \mathrm{m}$, showing a current of $\sim 70 \mu \mathrm{A}$, and the lower trace is the power in the full cell of the gun. The horizontal scale is $100 \mathrm{~ns} / \mathrm{div}$, and the vertical scale is $2 \mathrm{mV} / \mathrm{div}$ for the upper trace and $20 \mathrm{mV} /$ div for the lower trace.

\section{B. Quantum efficiency}

The first measurements of the photoelectron beam were made using a Faraday cup matched into $50 \Omega$. Taking the signal from the Faraday cup, the area under the signal voltage curve and Ohm's law can be used to determine the total charge in the beam. Figure 12 shows both the UV laser pulse that creates the electron bunch and the Faraday cup signal used to determine the charge. The fact that the laser appears to arrive after the electron beam is merely a result of different signal cable lengths; in addition, it is noted that the photodiode signal does not reflect the actual laser UV pulse duration. This particular measurement yields a total charge of $25 \mathrm{pC}$. Given the input energy of the UV laser as $6 \mu \mathrm{J}$, this yields a quantum efficiency for copper of $2 \times 10^{-5}$. The linearity of the bunch charge with the laser energy was verified experimentally between 0 and $25 \mathrm{pC}$. The aforementioned shot-to-shot variations occurring at $20 \mathrm{~Hz}$ can be partially eliminated by averaging over several shots; good results were obtained by averaging for $5 \mathrm{~s}$ over 100 shots. Furthermore, given the high rf field at the cathode and low laser energy used in the measurement, the beam current is not limited by space charge.

The second measurement performed with the Faraday cup was a study of the phase dependence of the charge. The rf phase relative to the laser was varied over $360^{\circ}$, which has two consequences: First, it affects the energy of the beam because it influences the timing of the acceleration of the charge; this means that, for most injection phases, there will be no beam because either the fields do not accelerate the electron bunch or accelerate it back into the photocathode. This effect is seen in the form of the data presented in Fig. 13. The super-Gaussian fit to the data is not chosen to calculate any beam parameters; instead, it merely represents a generic match to the data, which is

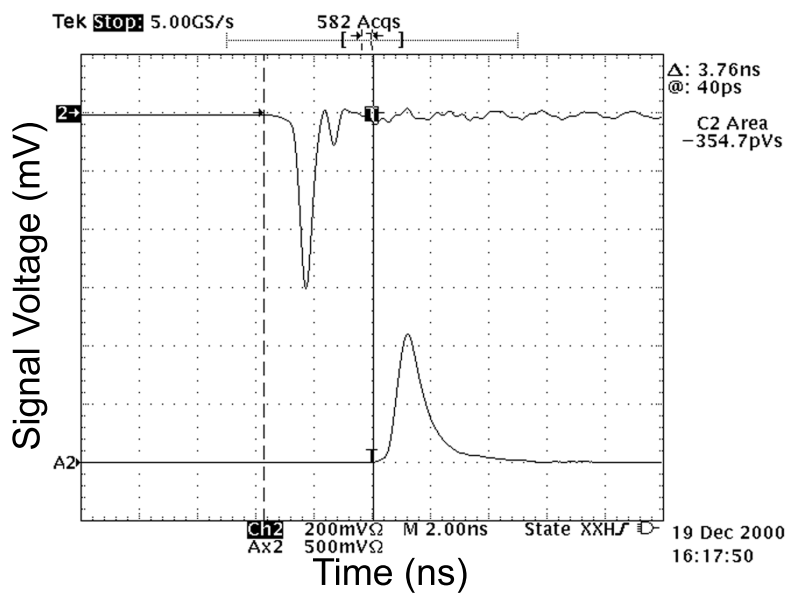

FIG. 12. Faraday cup measurement of the photoelectron current. The upper trace is the photoelectron signal from the Faraday cup, and the lower trace is the laser signal from a photodiode. The horizontal scale is $2 \mathrm{~ns} / \mathrm{div}$, and the vertical scale is $200 \mathrm{mV} /$ div for the upper trace and the $500 \mathrm{mV} / \mathrm{div}$ for the lower trace. This traces shows $7 \mathrm{pC}$ of charge. 


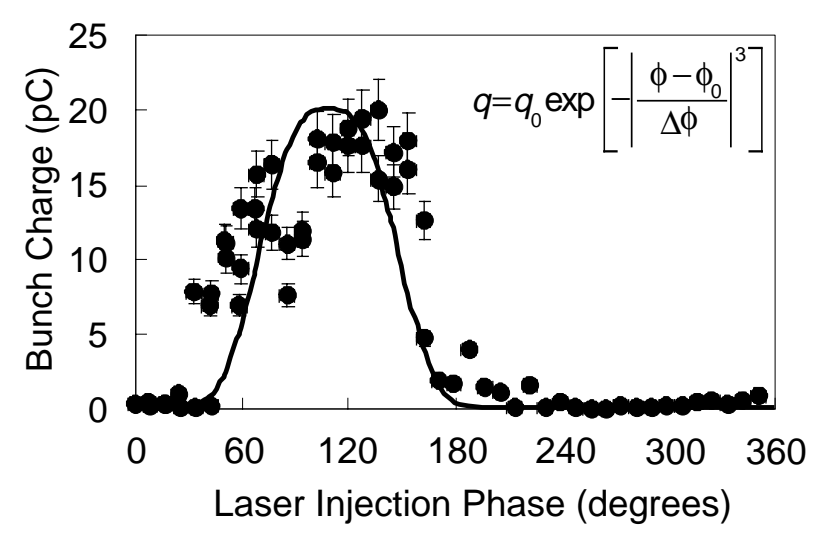

FIG. 13. Measured bunch charge versus rf phase when the laser is injected, showing the Schottky effect. The dots are the actual data, and the line is the fit to the super-Gaussian with equation shown. [This fit is used to match the shape of the data; compare with Fig. 17(b)].

used to compare to the shape of the coherent synchrotron data below. There is no particular physical motivation for the equation used. The second effect is that, by varying the phase, the electric field strength at the photocathode when the UV laser pulse arrives changes. A higher field results in an enhancement of the quantum efficiency (the Schottky effect). When the laser is synchronized with the peak of the rf cycle, more photoelectrons are produced and the final beam charge is higher. Although variation of the effective quantum efficiency with the rf phase is illustrated in Fig. 13, and the expected slope on the top of the curve is indicated, the large fluctuation in the data from the aforementioned instabilities prevents conclusive observation of the Schottky effect.

\section{Beam energy and energy spread}

To measure the energy and energy spread of the electron beam, an electron spectrometer [17] is used. The instability of the beam and the small (1 mm diameter) entry aperture on the spectrometer made coupling electrons into the device a challenging task. As a quick solution, a $2 \mathrm{~Hz}$ triangular wave was applied to one of the steering dipoles, creating a horizontal scan of the beam across the spectrometer aperture and ensuring that, on at least some shots, clean energy measurements could be recorded. A field of $0.73 \mathrm{kG}$ ( 3 A current) was used, and the image produced by the deflected electrons on the phosphor screen was recorded on Polaroid film. In order to analyze the beam profiles, one can convert the images from the streak camera into numerical data. Photographs taken by the streak camera are optically scanned at 600 dots per inch resolution into a computer and saved as eight-bit gray scale TIFF (tagged image file format) files. Since the streak camera optical settings are kept constant, the distances between reference points on the image, which correspond to portions of the beam seen by the camera, are known experimental pa- rameters. Hence, one can obtain a spatial scaling factor by directly measuring the distance between the reference points on the photographs and their equivalent dimensions in the experiment. Images saved in the TIFF format are not compressed, so the information contained in the original photograph is faithfully reproduced down to the limit of the scanner resolution. Also, image files are essentially arrays, where the row and column location of each array element correspond to the vertical and horizontal location of each pixel, and the value held by each element indicates the gray scale intensity of the pixel. These image files can be imported into the mathematical program suite MATHEMATICA from Wolfram Research, where the image array can be extracted and converted into a matrix using the graphics raster command. This matrix is then exported to a data file which is subsequently imported into the data analysis program AXUM from MathSoft, and each column, corresponding to a small time slice of data captured on the streak camera photographs, can be separately analyzed using the scaling factors as discussed above (Fig. 14). Using this technique, one can obtain highly accurate beamprofile data.

Using the magnetic field strength and the parameters given in [17], the energy of the beam was determined from the location of the image of the entrance slit to be $1.47 \mathrm{MeV}$, and the width of the spot gave a relative energy spread of $\Delta \gamma / \gamma_{0}=1.8 \pm 0.2 \%$ FWHM (see Fig. 14). The dispersion in the magnetic energy spectrometer can be evaluated as follows: the transverse velocity of the electrons is given by

$$
\nu_{\perp}=c \beta_{\perp}=\Omega_{\perp} r=\frac{e B_{\perp}}{\gamma m_{0}} r,
$$

where $B_{\perp}$ is the applied magnetic field and $r$ is the radius of the electron trajectory in the dipole magnet. Differentiating Eq. (2), and using the normalized momentum, we have

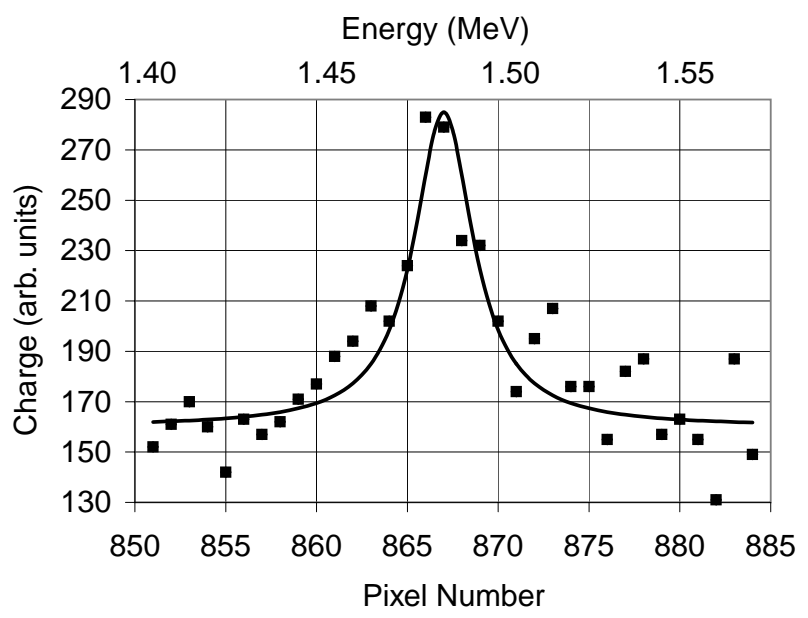

FIG. 14. Energy spread of the photoelectron beam as measured by the electron spectrometer. The energy spread is about $1.8 \%$. 


$$
\Delta u=\frac{\Delta p_{\perp}}{m_{0} c}=\Delta\left(\gamma \beta_{\perp}\right)=\frac{e B_{\perp}}{m_{0} c} \Delta r
$$

for a field of $0.73 \mathrm{kG}$, the dispersion is $42.7 \mathrm{~m}^{-1}$. As the beam energy is measured at $1.47 \mathrm{MeV}$, and a pixel on the recorded energy spectrum corresponds to $0.4 \mathrm{~mm}$, we find that the four-pixel FWHM spectrum has a momentum spread $\Delta u=0.068$ which corresponds to a relative energy spread $\Delta \gamma / \gamma=1.8 \%$, since $\gamma^{2}=1+u^{2}$.

\section{Beam emittance}

To measure the beam emittance, direct examination of the beam divergence is performed. First, a phosphor screen was used to determine the size of the electron beam [Fig. 15(a)], which was found to be $1.68 \mathrm{~mm}$ in diameter. This screen is then replaced by a thin tantalum (Ta) plate, with three holes of diameter $0.25,0.5$, and $1 \mathrm{~mm}$. Placing this plate in the beam path will completely
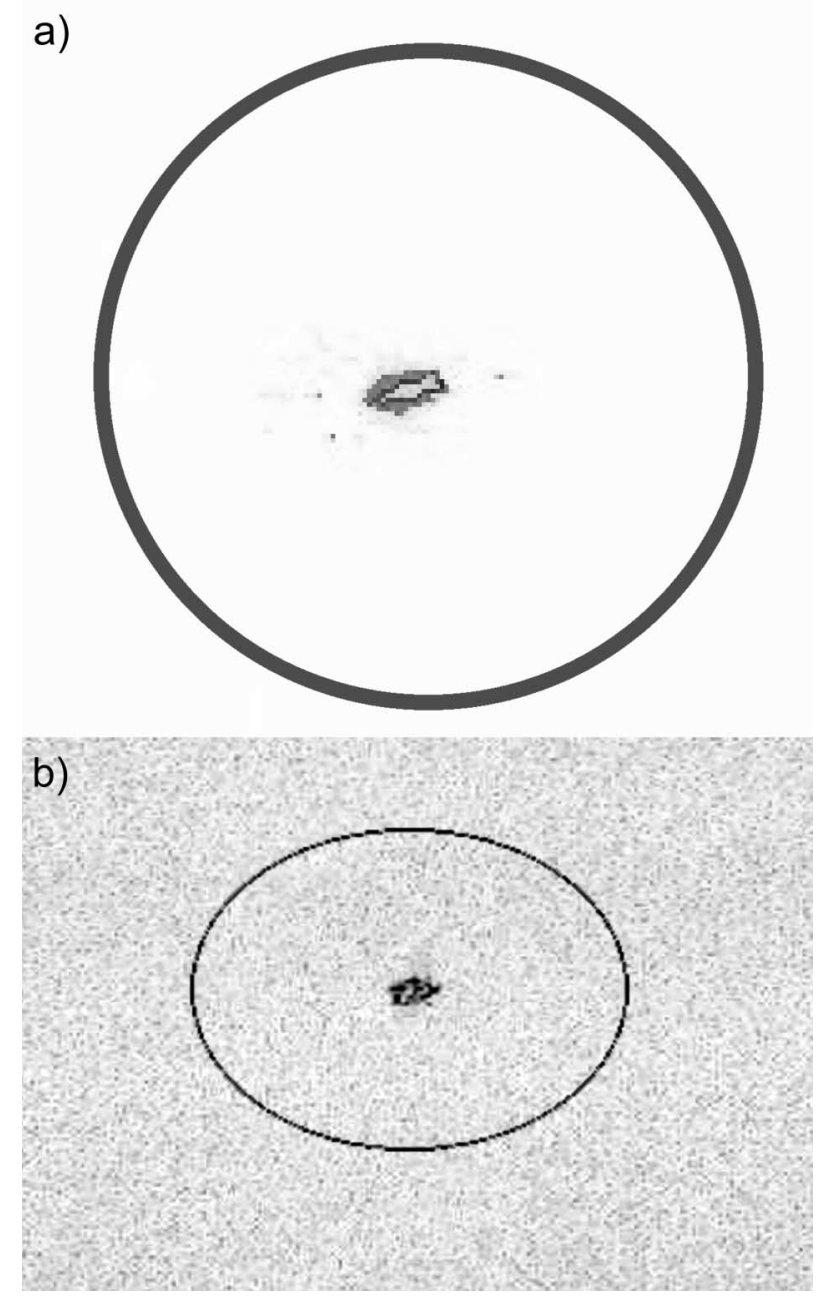

FIG. 15. Images of the phosphor screen taken (a) at the location where the Ta aperture is placed and (b) a meter downstream from the Ta aperture. The circles represent the edge of the phosphor screen which is $19 \mathrm{~mm}$ in diameter. stop the beam. The $1 \mathrm{~mm}$ hole is centered on the beam to allow a small sample of the electron beam, with known diameter, to pass. The phosphor screen is placed $0.997 \mathrm{~m}$ away from the Ta plate. The size of the electron beam at the phosphor is measured to be $1.84 \mathrm{~mm}$, as shown in Fig. 15(b). Because the spot started with a diameter of $1 \mathrm{~mm}$ from the hole in the Ta, this means the edge divergence of the beam is $0.84 \mathrm{mrad}$, while the maximum core divergence, for on-axis electrons, is $1.84 \mathrm{mrad}$. Since the charge of this bunch is sufficiently small, space-charge effects on the emittance are negligible, and this divergence is a direct reflection of the transverse momentum spread of the beam. The transverse phase space of the beam can then be mapped by moving the $\mathrm{Ta}$ aperture and measuring the corresponding divergence angles. Using this technique, and limiting the count to $90 \%$ of the bunch charge, the normalized emittance of the beam is found to be $\varepsilon_{n} \approx 1.65 \pi \mathrm{mm} \mathrm{mrad}$. This is an excellent number, especially in view of the relatively low beam energy and absence of emittance compensation; furthermore, it is in good agreement with PARMELA simulations as presented in Table I.

\section{E. Bunch duration measurements}

To get an estimate of the bunch length, a streak camera operating at its maximum streak speed of $20 \mathrm{ps} / \mathrm{mm}$ is used to image the phosphor screen. The streak image is captured on Polaroid film, which is then scanned into a computer, as discussed in Sec. III C. To determine the resolution of the camera, a measurement of incident UV laser pulses was first made [Fig. 16(a)]. The streak image was measured to be 2 ps long FWHM, which matches the nominal resolution of the camera, as the actual duration of the UV laser pulse is well under 100 fs [see Fig. 8(a)]. When the electron beam is present, a prompt emission is observed on the streak image, followed by an image of the slow fluorescence of the phosphor. To distinguish between the light emitted by the electrons and that corresponding to the diffused light from a UV laser pulse striking the photocathode, a Plexiglas filter is used to cover half of the entrance slit: only the visible optical radiation produced by the electrons propagates through the filter, while the laser light is strongly attenuated; in addition, the delay induced by the refractive index of the Plexiglas can be used to further calibrate the streak camera. It is unclear if the observed line is purely Čerenkov radiation, or is also produced by fast $\mathrm{x}$ rays interacting with the phosphor screen, but the duration of the light pulse in either case is directly correlated to the duration of the electron bunch, as shown in Fig. 16(b), where we find it to be equal to the 2 ps resolution of the camera itself, as determined by the technique described above; this indicates that the duration of the electron bunch is less than 2 ps. Further evidence of the extremely short electron bunch duration is obtained by considering the coherent synchrotron radiation emitted by 

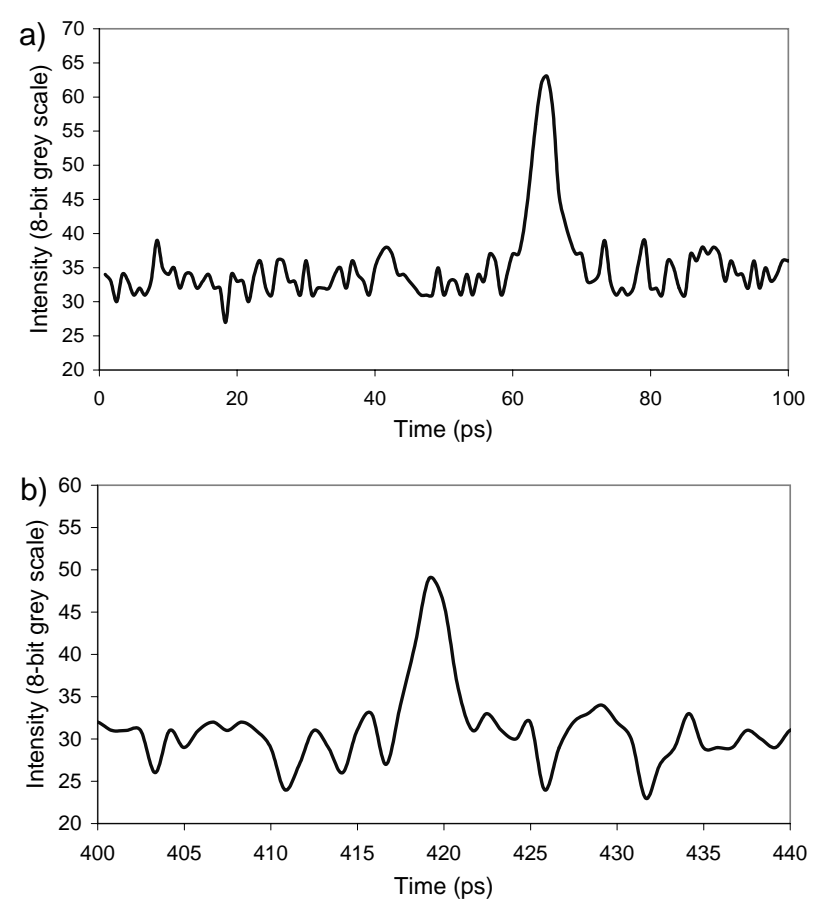

FIG. 16. Streak camera image line outs. (a) The reflection of the laser beam off the photocathode with no rf in the gun (and therefore no photoelectron beam and emission produced). The 2 ps length demonstrates the camera resolution. (b) Emission from the photoelectron beam with the laser blocked by a filter, showing the duration to be at or below the camera resolution (2 ps).

the electrons when propagating in a short, slow-wave structure; this is studied in greater detail in Sec. III G.

\section{F. Timing jitter}

We studied the timing jitter between the rf fields in the photoinjector, the UV laser pulses, and the photoelectrons. The measurement of the charge produced as a function of the laser injection phase provides an indirect estimate of the timing jitter between the UV laser pulses on the photocathode and the rf fields exciting the accelerator structure: If the jitter is larger than approximately 10 degrees of rf phase, such a measurement could not be performed; in fact, the good contrast on the coherent synchrotron radiation curves [Fig. 17(b)] shows that the transition from photoemission to dark current can only be pinpointed to within 10 degrees.

To obtain a better estimate, we have systematically measured the timing between UV laser pulses and the visible radiation flashes produced by the photoelectrons on the screen used in Sec. IIID; this is achieved by acquiring a large number of shots and using statistical tools to analyze the experimental results. The first calibration is obtained by carefully measuring the timing jitter between the streak camera and the incident UV laser pulses; unfortunately, that number is found to be high, of the order of $60 \mathrm{ps}$. This large number is due to jitter in the oscilloscope, the
SRS box, and mostly jitter inherent in the streak camera. The reason for requiring a low base number is that, for uncorrelated sources of timing jitter, the statistical width of the measured distribution scales as

$$
\Delta t=\sqrt{\Delta t_{1}^{2}+\Delta t_{2}^{2}+\cdots+\Delta t_{n}^{2}},
$$

where the $\Delta t_{i}$ correspond to different mechanisms producing timing jitter in the system. For our experiments, the main known and measured values are $\Delta t_{l}<2$ ps for the measured laser pulse duration, as streaked by the camera, $\Delta t_{e}<2 \mathrm{ps}$, for the optical light flashes produced by the photoelectron beam, and $\Delta t_{l s}=60 \mathrm{ps}$ is the timing jitter measured between the streak camera and the UV laser pulses. Clearly, this last contribution dominates in Eq. (4) and limits the accuracy of our measurement of the timing jitter between the photoelectrons and the incident UV laser pulses: If the width of the Gaussian statistical distribution can be estimated to within a relative accuracy $\varepsilon$, it is easily seen that the corresponding precision on the timing jitter will be of the order of $\sqrt{2 \varepsilon}$. In our case, $\varepsilon \approx 5 \%$, and the minimum value of $\Delta t_{l e}$ that can be measured reliably is approximately equal to $\sqrt{2 \varepsilon} \Delta t_{l s}=19 \mathrm{ps}$. Our analysis shows that the width of both distributions, the UV laser pulses and the Čerenkov radiation flashes, are comparable; therefore, we conclude that the timing jitter between the laser and electron beams is less than 20 ps. This is an upper limit, and the actual value is most likely much less, since 20 ps corresponds to around 60 degrees of rf phase. For more accurate measurements, one would need to compare single-event pairs, but the amount of light produced by the electrons is quite small, making such a precise measurement a much more challenging task.

\section{G. Coherent synchrotron radiation}

As mentioned earlier, the photoelectron bunches produced by the $X$-band rf gun are very short, and streak camera measurements put an upper limit of approximately 2 ps on their duration; thus, they can radiate coherently at short wavelengths. This effect has been previously observed [18]. We performed experiments where the bunches propagate through a corrugated waveguide and couple to the slow waves supported by that structure. To demonstrate that the radiation produced is, indeed, coherent, we have measured the power radiated as a function of the bunch charge. The variation of the bunch charge can be achieved in two different manners: On the one hand, the laser injection phase can be fixed, and the UV energy can be changed by using different combinations of calibrated neutral density filters, as shown in Fig. 17(a); on the other hand, the UV energy can be fixed, and the laser injection phase varied, thus taking advantage of the charge variation with phase, as studied in Sec. III A and shown in Fig. 17(b).

The first experimental measurements consisted of measuring the rf power radiated by the bunches. A coupling 

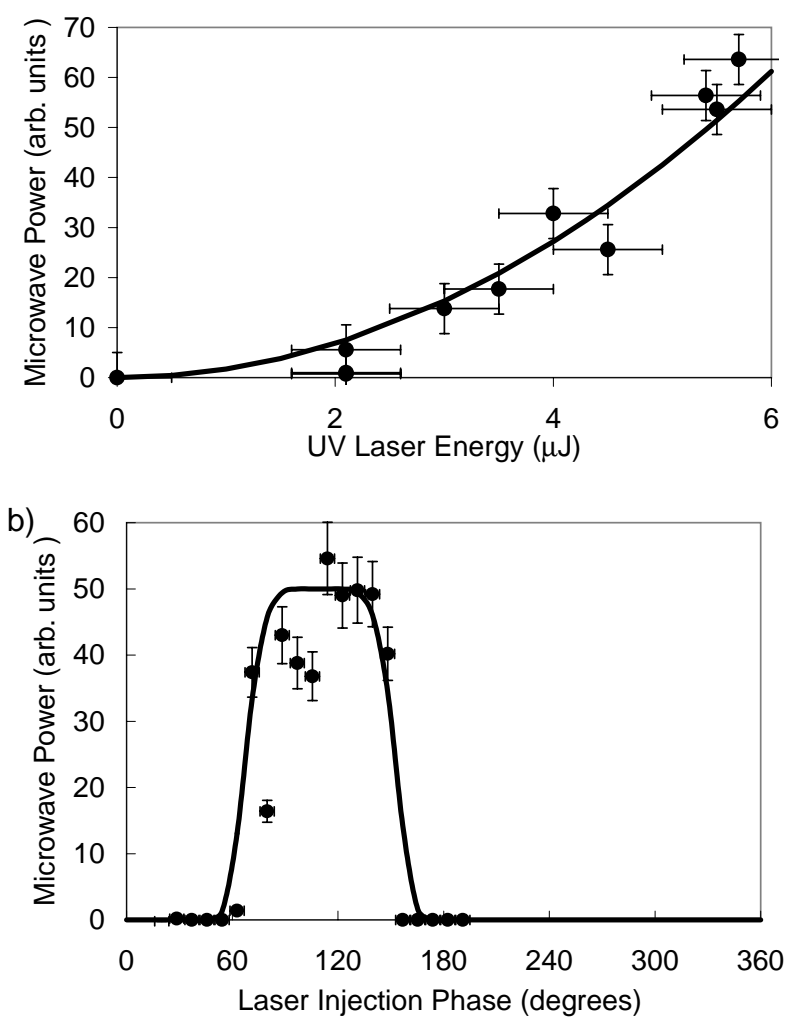

FIG. 17. Coherent synchrotron radiation measurements: (a) radiation intensity as a function of laser energy with a quadratic fit, and (b) radiation intensity as a function of $\mathrm{rf}$ phase relative to laser injection. The fit in (b) is the equation resulting in the fit of Fig. 13 squared (only the total amplitude was varied, not the width).

horn gathered the radiation from the end of the beam line passing through a quartz window, and a waveguide section (colinear with the beam line) was used to provide low frequency cutoff before the wave reached the different frequency detectors used. We were able to confirm the emission of extremely short pulses of rf power up to Ka-band; at higher frequencies, the detector speed is believed to be insufficient: the characteristic time scale of the radiation bursts is given by the slippage between the photoelectron bunch and the radiation pulse; as the corrugated waveguide section was only $10 \mathrm{~cm}$ long, the pulses were estimated to be $50-500 \mathrm{ps}$ in duration, or approximately $2-20 \mathrm{rf}$ cycles. At low frequency, between $X$-band and Ka-band, we were able to measure pulses with a FWHM of 500 ps, as illustrated in Fig. 18; this is to be compared with the detector rise time, specified at $<600 \mathrm{ps}$. At higher frequencies, the detectors are not fast enough to follow the pulses, and we are considering the possibility of using broadband mixers to perform more detailed measurements.

The quadratic scaling of the power radiated versus the bunch charge is very clearly demonstrated in Fig. 17(a); furthermore, we can take the super-Gaussian fit from Fig. 14, properly squared, and match the rf data: The agreement is good [see Fig. 17(b)] and confirms the

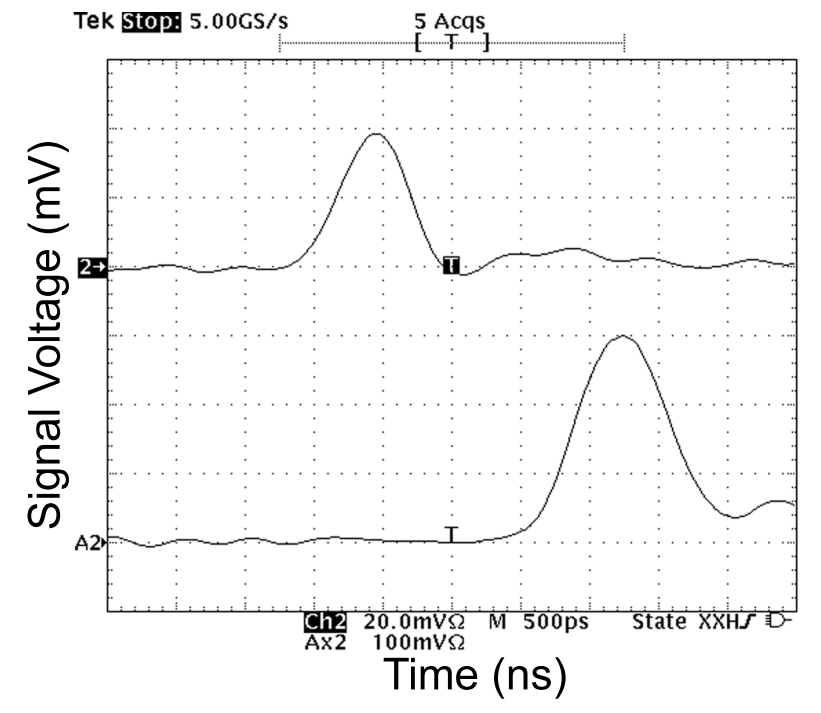

FIG. 18. Coherent synchrotron radiation measurements. The upper trace shows the signal from an $X$-band microwave detector placed at the end of the beam line, and the lower trace shows the laser photodiode signal. This demonstrates the shortness of the microwave radiation burst (below the response time of the oscilloscope). The horizontal scale is $500 \mathrm{ps} / \mathrm{div}$, and the vertical scale is $20 \mathrm{mV} /$ div for the upper trace and $100 \mathrm{mV} / \mathrm{div}$ for the lower trace.

coherence of the radiation emitted by the bunches. This has important potential application to millimeter wave and far infrared coherent radiation generation, as the electron beams produced by the compact rf photoinjector are fully prebunched and can be radiated coherently up to the $\mathrm{THz}$ spectral region. In particular, our group produced extensive theoretical work on prebunched free-electron masers [6] and the theory of coherence in synchrotron radiation [19] and showed that ultrawideband, chirped-pulse radiation could be produced at power levels in excess of $2 \mathrm{MW}$, in the 100-200 GHz range. Such devices could be extremely useful for advanced radar systems and as tools to probe materials and plasmas.

Finally, these measurements provide indirect confirmation that the electron bunches produced by our $X$-band photoinjector are very short. They also indicate, as pointed out by Carlsten [20], that one must pay very close attention to the beam line design for a system using this technology, as coherent synchrotron radiation typically degrades the high-brightness bunches because of correlated recoil effects. This implies that one must avoid bends, bellows, and cavities, which all strongly couple to the photoelectron bunches, and instead strive to provide smooth, continuous transverse boundaries for the beam line, in order to minimize unwanted radiation. Furthermore, the beam line can also be designed to avoid trapped modes and provide quenching of the lower frequency modes by a judicious combination of polarization and cutoff frequencies for those modes that couple most strongly with the prebunched beam. 


\section{CONCLUSIONS}

An $X$-band $(8.574 \mathrm{GHz})$ rf photoinjector was successfully operated, creating a reproducible $1.5-2 \mathrm{MeV}$ electron beam with low (1.8\%) energy spread, good $(1.65 \pi \mathrm{mm} \mathrm{mrad})$ emittance, and short $(<2 \mathrm{ps})$ photoelectron bunches. Coherent synchrotron radiation was seen to be emitted by the electron beam as it propagated along the beam line. To our knowledge, this represents the first experimental study of coherent synchrotron radiation in a high-frequency photoinjector. The instabilities observed in the beam demonstrate the need for very careful control of laser pointing, energy, and pulse-length stability to produce a reliable high-quality beam.

\section{ACKNOWLEDGMENTS}

This work was performed under the auspices of the U.S. Department of Energy by the University of California, Lawrence Livermore National Laboratory, partially through the Institute for Laser Science and Applications, under Contract No. W-7405-Eng-48. Additional funding was provided by AFOSR Grant No. F4962099-1-0297 and NIH Contract No. N01-CO-97113. One of us (A. L. T.) thanks Professor T. R. Reed for insightful discussion concerning digital image processing.

[1] D. T. Palmer, X. J. Wang, R. H. Miller, M. Babzien, I. Ben-Zvi, C. Pellegrini, J. Sheehan, J. Skaritka, H. Winick, M. Woodle, and V. Yakimenko, in Proceedings of the 1997 Particle Accelerator Conference, Vancouver, Canada, edited by M. Comyn, M. K. Craddock, M. Reiser, and J. Thomson (IEEE, Piscataway, NJ, 1998), p. 2687.

[2] R. Alley, V. Bharadwaj, J. Clendenin, P. Emma, A. Fisher, J. Frisch, T. Kotseroglou, R. H. Miller, D. T. Palmer, J. Schmerge, J. C. Sheppard, M. Woodley, A. D. Yeremian, J. Rosenzweig, D. D. Meyerhofer, and L. Serafini, Nucl. Instrum. Methods Phys. Res., Sect. A 429, 324 (1999).

[3] D. Yu, T. Lee, S. Rajagopalan, and J. Chen, in Proceedings of the 1997 Particle Accelerator Conference, Vancouver, Canada (Ref. [1]), p. 2802.
[4] W. J. Brown, S. Trotz, K. E. Kreischer, M. Pedrozzi, M. A. Shapiro, and R. J. Tempkin, Nucl. Instrum. Methods Phys. Res., Sect. A 425, 441 (1999).

[5] F. B. Kiewiet, O. J. Luiten, G. J. H. Brussaard, J. I. M. Botman, and M.J. Van Der Wiel, in Proceedings of the 7th European Particle Accelerator Conference, 2000, Vienna, Austria, http://accelconf.web.cern.ch/AccelConf/ e00/index.html, p. 1660.

[6] G. P. Le Sage, H. X. C. Feng, L. L. Laurent, F. V. Hartemann, N.C. Luhmann, S. N. Fochs, M.D. Perry, G. A. Westenskow, and J.P. Heritage, IEEE Trans. Plasma Sci. 24, 781 (1996).

[7] A. T. Amatuni and M. L. Petrossian, Nucl. Instrum. Methods Phys. Res., Sect. A 455, 128 (2000).

[8] J. L. Martin, Curr. Med. Chem. 3, 419 (1996).

[9] F. V. Hartemann, Phys. Plasmas 5, 2037 (1998).

[10] Y. J. Chen, Nucl. Instrum. Methods Phys. Res., Sect. A 279, 433 (1989).

[11] J. Rosenzweig and E. Colby, in Advanced Accelerator Concepts: Sixth Annual Conference, edited by P. Schoessow, AIP Conf. Proc. No. 335 (AIP, New York, 1995), p. 724.

[12] M. A. Allen, R. S. Callin, H. Deruyer, K. R. Eppley, W. R. Fowkes, W. B. Herrmannsfeldt, T. Higo, H. A. Hoag, T. L. Lavine, T. G. Lee, G. A. Loew, R.H. Miller, P. L. Morton, R. B. Palmer, J. M. Paterson, R. D. Ruth, H.D. Schwarz, Y. Takeuchi, A.E. Vlieks, J.W. Wang, and P. B. Wilson, Stanford Linear Accelerator Center Report No. SLAC-PUB-4650, 1988.

[13] G. P. Le Sage, C. V. Bennett, W. E. White, E. C. Landahl, L. L. Laurent, N. C. Luhmann, Jr., F. V. Hartemann, C. H. Ho, W. K. Lau, and T. T. Yang, Phys. Plasmas 5, 2048 (1998).

[14] C. Spielmann, P. F. Curley, T. Brabec, and F. Krausz, IEEE J. Quantum Electron. 30, 1100 (1994).

[15] A. Stingl, M. Lenzner, C. Spielmann, F. Krausz, and R. Szipocs, Opt. Lett. 20, 602 (1995).

[16] R. H. Fowler and L. Nordheim, Proc. R. Soc. London A 119, 173 (1928).

[17] B. Kulke, M.J. Burns, and T.J. Orzechowski, Rev. Sci. Instrum. 58, 1153 (1987).

[18] T. Nakazato, M. Oyamada, N. Niimura, S. Urasawa, O. Konno, A. Kagaya, R. Kato, T. Kamiyama, Y. Torizuka, T. Nanba, Y. Kondo, Y. Shibata, K. Ishi, T. Ohsaka, and M. Ikezawa, Phys. Rev. Lett. 63, 1245 (1989).

[19] F. V. Hartemann, Phys. Rev. E 61, 972 (2000).

[20] B. E. Carlsten, Phys. Rev. E 54, 838 (1996). 